TECHNICAL NOTE

K.E. Emblem

A. Bjornerud

\section{An Automatic Procedure for Normalization of Cerebral Blood Volume Maps in Dynamic Susceptibility Contrast-Based Glioma Imaging}

SUMMARY: To characterize gliomas from dynamic susceptibility contrast (DSC)-based cerebral blood volume (CBV) maps, a CBV value from a normal-appearing region of interest is typically identified manually and used to normalize the CBV maps. This method is user-dependent and time-consuming. We propose an alternative approach based on automatic identification of normal-appearing first-pass curves from brain tissue. Our results in 101 patients suggest similar or better diagnostic accuracy values than the manual approach.
$\mathbf{T}$ he correlation between histopathologic glioma malignancy and cerebral blood volume (CBV) derived from dynamic susceptibility contrast (DSC) imaging is well known. ${ }^{1}$ Using either a hot-spot ${ }^{2}$ or a histogram analysis method, ${ }^{3,4}$ one typically assesses glioma malignancy from relative CBV $(\mathrm{rCBV})$ values in the tumor area following normalization of quantitative $\mathrm{CBV}$ values to a reference normal-appearing mean CBV value. Typically, normal-appearing white matter areas are used as the basis for the reference CBV value, ${ }^{2}$ though alternative methods exist using white matter for tumors with their origin in the white matter and gray matter for tumors with their origin in the gray matter. ${ }^{4}$ The current method for a manual selection of the reference tissue is time-consuming. Furthermore, the reference region of interest is defined by a few pixels only, with resulting user dependence and lack of robustness. Alternatively, using a histogram approach to automatically identify a set of voxels in the first precontrast DSC image with intensity values corresponding to normal brain tissue has been suggested. ${ }^{5}$ Although promising, the use of a single baseline image renders the method MR imaging system-dependent and prone to saturation effects. In addition, no knowledge of the dynamic time curve is included in the analysis.

We propose an alternative automatic technique using the DSC first-pass response to derive a section-specific mean reference $\mathrm{CBV}$ value from all pixels determined to represent normal-appearing brain tissue. With the characteristics of the first-pass response, confounding pixels thought to represent vessels, noise, tumor, or nonbrain pixels are automatically excluded from the reference CBV value, and the resulting mean $\mathrm{CBV}$ value is therefore expected to represent normal-appearing gray and white matter values.

Received March 9, 2009; accepted after revision April 17.

From the Departments of Medical Physics (K.E.E., A.B.) and Interventional Center (K.E.E), Rikshospitalet, Oslo University Hospital, Oslo, Norway; and Department of Physics (A.B.), University of Oslo, Oslo, Norway.

This work was supported by a grant from the Norwegian Research Council (K.E.E.).

Please address correspondence to Kyrre E. Emblem, MSc, Department of Medical Physics, Rikshospitalet, Oslo University Hospital, Sognsvannsveien 20, N-0027 Oslo, Norway; e-mail: kyrre.eeg.emblem@rikshospitalet.no

Indicates open access to non-subscribers at www.ajnr.org

DOI 10.3174/ajnr.A1680

\section{Technique}

\section{Patients}

Study approval was obtained from the Regional Medical Ethics Committee, and patients were included only if informed consent was signed. The proposed technique was evaluated in 101 patients (6-76 years of age; mean age, 46 years; 57 males, 44 females) with a histopathologic diagnosis of a glioma after MR imaging and subsequent surgery. Fifteen patients had recurrent tumors. No patients received radiation treatment before MR imaging.

\section{MR Imaging}

MR imaging was performed at $1.5 \mathrm{~T}$ using an 8-channel head-coil (Symphony/Sonata; Siemens, Erlangen, Germany) or a 12-channel head-coil (Avanto; Siemens). The protocol included a 19-section axial T2-weighted fast spin-echo sequence (TR/TE, 4000/104 ms) and a pre-/postcontrast 19-section axial T1-weighted spin-echo sequence (TR/TE, 500/7.7 ms). DSC imaging was performed by using a wholebrain (12-14 sections) single-shot gradient-echo echo-planar imaging sequence (TR/TE, 1430/46 ms) acquired during contrast agent administration. The voxel size was $1.80 \times 1.80 \times 5 \mathrm{~mm}^{3}$, with an intersection gap of $1.5 \mathrm{~mm}$ and a flip angle of $90^{\circ}$. For each section, 50 images were recorded at each TR. After approximately 8 time points, $0.2 \mathrm{mmol} / \mathrm{kg}$ of gadobutrol (Gadovist; Bayer Schering Pharma, Berlin, Germany) was injected intravenously.

\section{Normalization Procedure}

First, by using the first precontrast DSC image only, noisy pixels with a mean baseline value below a noise threshold were identified and excluded by using the Otsu method. ${ }^{6}$ Also, tumor pixels were identified and excluded by performing a 5-class k-means clustering on the first precontrast DSC image and removing the cluster class with the highest mean signal-intensity value. ${ }^{7}$

Second, in all DSC sections and for all remaining pixels, an exhaustive search was performed to identify automatically first-pass curves thought to represent normal-appearing brain tumor pixels. The identification procedure was performed by excluding all confounding vessel-like and otherwise abnormal first-pass curves by using the following criteria:

- Vessel pixels were identified and excluded by using a previously described DSC-based vessel-segmentation technique. ${ }^{7}$

- Spur pixel values from first-pass curves with a peak value in the global baseline area (ie, before primary injection) were identified and excluded.

- Erroneous pixels from first-pass curves with evident T1-leakage 

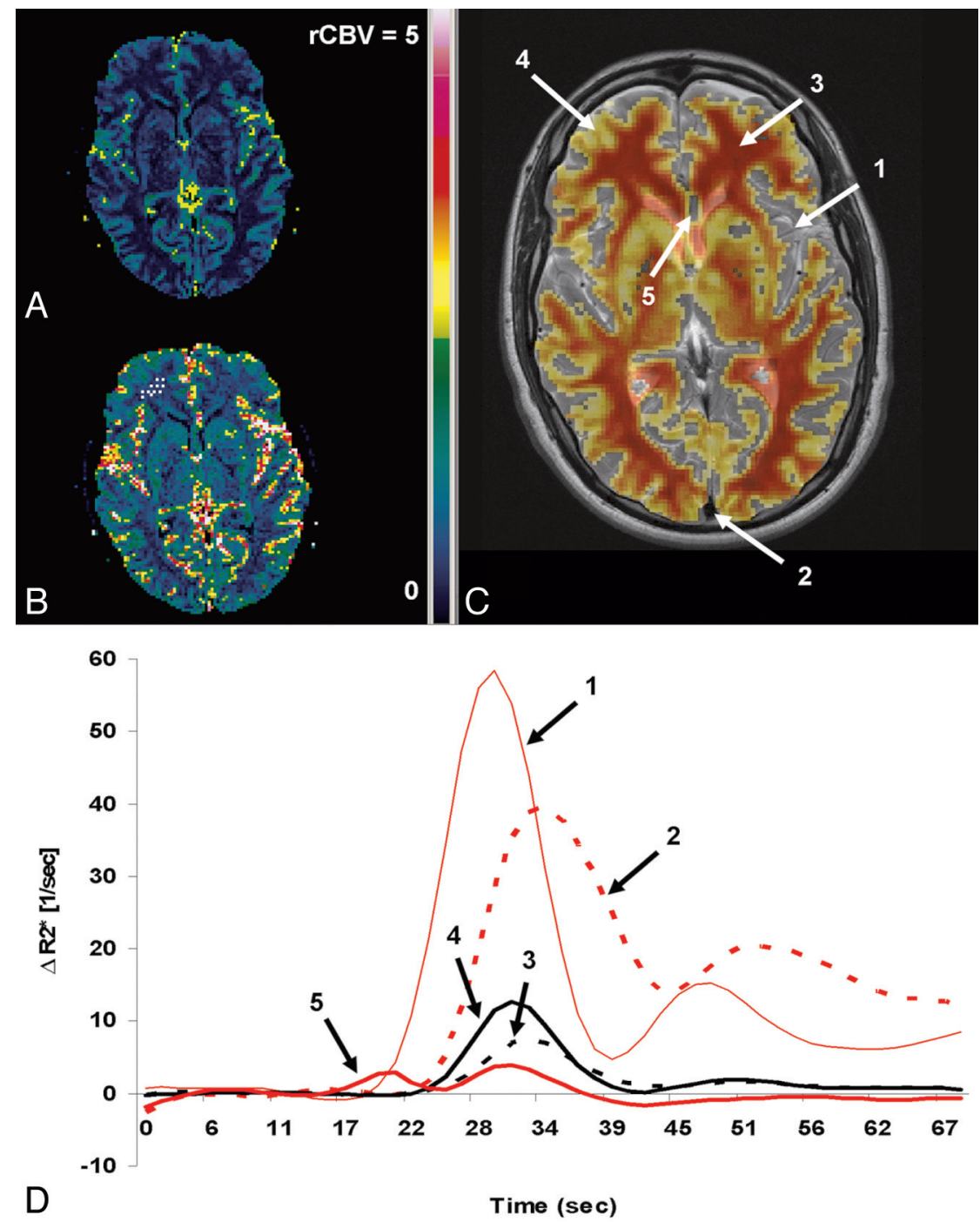

Fig 1. $A$ and $B$, Axial normalized rCBV maps using the automated method $(A)$ and the manual reference method $(B)$. $A$ and $C$, The reference CBV value $(A)$ is a mean value of CBV values from normal-appearing white matter and gray matter tissue, shown as an overlay (red/yellow) on an axial T2-weighted MR image (C). $B$, Only white matter CBV values are used to derive the reference CBV value (shown as white scatter points in the right frontal lobe), resulting in higher normalized rCBV values compared with $A$. $D$, The first-pass curves from arteries (1), veins (2), white matter (3), gray matter (4), and a noisy pixel $(5)$ are shown. $C$, First-pass curves with characteristics similar to the red curves $(1,2,5)$ are automatically excluded from the normal-appearing CBV values, to derive the automatic reference CBV value. Sec indicates second.

effects (postbolus signal intensity below baseline) or T2-leakage effects (postbolus signal intensity $>2$ SDs above baseline) compared with a mean global first-pass curve were identified and excluded. ${ }^{1}$

Examples of included and excluded first-pass curves are shown in Fig 1. Finally, a mean reference CBV value for each DSC section was assessed from the remaining pixels thought to represent normal-appearing brain tissue.

\section{DSC Analysis}

The CBV maps were generated by using established methods and corrected for possible extravascular contrast agent leakage effects. ${ }^{2,3,8}$ Two sets of rCBV maps were created by normalizing all CBV pixels to the following: 1) the automatically defined reference CBV value, and 2) a conventional manually defined CBV reference value in normal-appearing white matter (Fig 1). ${ }^{2}$ As described by others, on the basis of anatomic MR underlay images and CBV overlay maps before any $\mathrm{rCBV}$ normalization, tumor regions of interest were drawn manually in each section by an experienced neuroradiologist, taking care to avoid areas of necrosis, edema, or nontumor macrovessels evident on the postcontrast T1-weighted images. ${ }^{3,8}$ All image processing was performed by using nordicICE (NordicImagingLab, Bergen, Norway) and Matlab R2008a (MathWorks, Natick, Mass).

\section{Statistical Comparisons}

The time used to perform the conventional and automatic normalization procedure was recorded. Furthermore, a histogram analysis $\operatorname{method}^{3}$ was used to derive pair-wise correlation measures (Pearson) between histogram peak heights, and logistic regression was used in combination with available survival data to derive Cox hazard ratios and Kaplan-Meier survival curves with respect to separating a highrisk group (survival $<2$ years after MR imaging examination) from a low-risk group ( $>2$ years) regardless of histopathologic grade. In the 


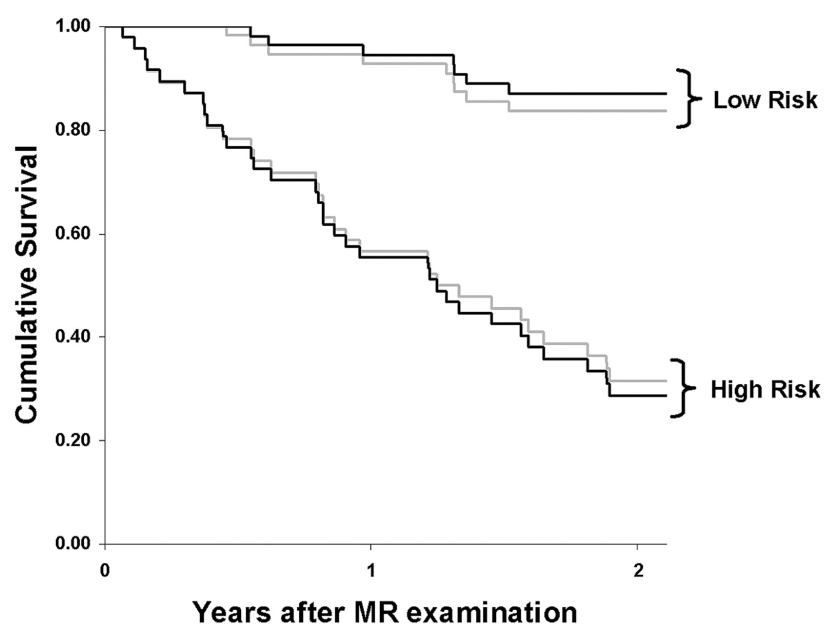

Fig 2. On the basis of histogram peak heights with patient age as a covariate, the figure shows the results of Kaplan-Meier survival analysis for the automatic normalization procedure (black lines) and the manual reference method (gray lines) when separating a high-risk group with expected survival of $<2$ years from a low-risk group with expected survival of $>2$ years. The logrank value between the high- and low-risk groups was higher for the automatic method (Mantel-Cox $\chi^{2}=38.287 ; P<.001$ ) compared with the manual reference method $(30.029 ; P<.001)$.

regression analysis, patient age was included as a second covariate. Statistical analysis was performed by using the Statistical Package for the Social Sciences, Version 15 (SPSS, Chicago, Ill).

\section{Results}

Of the 101 patients investigated, there were $6 \mathrm{WHO}$ grade I pilocytic astrocytomas, 20 grade II diffuse astrocytomas, 9 grade II oligodendrogliomas, 15 grade II oligoastrocytomas, 1 grade II ganglioglioma, 3 grade III anaplastic astrocytomas, 3 grade III anaplastic oligodendrogliomas, 6 grade III anaplastic oligoastrocytomas, and 38 grade IV glioblastomas.

Based on the manually defined white matter definitions, the mean rCBV values in normal-appearing white matter were $0.96 \pm .11$ for the manual method and $0.64 \pm .09$ for the automatic method (Fig 1). The manual procedure took approximately 1-2 minutes to perform, whereas the automatic procedure was performed within seconds.

The pair-wise correlation between the histogram peak heights when using the manual reference method and the automatic normalization procedure was significant at the 0.01 level $(r=0.792)$. The logrank (Mantel-Cox $\left.\chi^{2}\right)$ values describing the difference between the survival curves for the low- and high-risk groups were higher for the automatic method than for the manual reference method $(38.287 ; P<.001$ and $30.029 ; P<.001$, respectively) as shown in Fig 2 . The corresponding Cox hazard ratios were 8.707 (95\% confidence interval [CI], 3.834-19.774) and 6.229 (95\% C I, 2.955-13.131) for the automatic and manual methods, respectively.

\section{Discussion}

Current methods for manual selection of reference CBV values in DSC-based glioma grading are time-consuming and subjective. ${ }^{2-4}$ In any DSC-based grading method not using quantitative CBV maps after determination of an arterial input function, the normalization procedure is a critical step providing relative CBV values comparable between patients. Also, the normalization procedure corrects for signal intensity-to-noise variations across the image volume. In our study, we propose a fast automatic method including every image pixel in the CBV maps with a firstpass curve suggestive of normal-appearing brain tissue. Pixel values thought to represent arteries and veins were excluded. In addition, because the applied vessel-segmentation routine only identifies pixels with indisputable vessel-like first-pass curves, the normalization procedure was further improved by excluding presumed tumor pixels and pixels with noisy first-pass curves. Although the proposed normalization procedure was compared with the manual reference normalization from 1 neuroradiologist only and thus should also be compared with the inter- and intraobserver variations of the manual reference method, our results suggest that the automatic normalization procedure correlates well with the manual reference method. ${ }^{3}$

Results of the Cox proportional hazards model and Kaplan-Meier survival analysis suggest that the automatic method provides better discrimination between a high-risk and a low-risk patient group. Instead of assessing diagnostic accuracy values with respect to glioma grade, survival analysis was used because a histopathologic glioma grade from tissue sampling is inherently limited by small and inaccurate tissue samples, inaccessibility, and subjective grading criteria. Potentially, in addition to survival analysis, the proposed normalization procedure is amendable to related applications such as differentiation of radiation treatment effects from recurrent tumor.

The proposed normalization procedure excluded image areas identified as tumor by the automatic k-means-clustering premask on the first prebolus DSC image. ${ }^{5,7}$ Although this image is prone to image artifacts as described in our study, this source of error should be limited because the first prebolus DSC image was used to exclude areas of T2 abnormality, secondary to pathology or fluids only. Furthermore, our method assumes the presence of tumor tissue in at least $\geq 1$ DSC image section. Thus, if one is performing the normalization procedure on nontumor DSC images, the k-mean-clustering premasking routine can be removed. Also, because the tumor may be located in both the right and left hemispheres, the mean reference CBV values were based on normal-appearing voxels from the entire brain instead of just the contralateral side.

The inclusion of both gray and white matter CBV values resulted in lower mean reference $\mathrm{CBV}$ values for the automatic method compared with the manual reference method, by using white matter only. Thus, as gliomas involve predominately white matter, the automatic procedure may underestimate tumoral CBV values in predominantly white matter lesions. However, with the use of an intermediate gray and white mean $\mathrm{CBV}$ value and the large quantity of tumoral CBV values included in the histogram analysis, this source of error should be minimal. Equivalently, the manual reference method, by using white matter values only, always overestimates gray matter lesions.

In conclusion, we propose a rapid user-independent method for normalizing rCBV maps in DSC-based glioma imaging. The proposed method provides similar or better diagnostic accuracy values than the current manual approach and may easily be implemented in the clinical routine directly on 
the MR imaging scanner or in third-party clinical applications in the hospital-wide PACS system.

\section{Acknowledgments}

We thank Drs Paulina Due-Tonnessen, David Scheie, and John K. Hald, $\mathrm{PhD}$, Rikshospitalet, Oslo University Hospital, for contributions to this work.

\section{References}

1. Paulson ES, Schmainda KM. Comparison of dynamic susceptibility-weighted contrast-enhanced MR methods: recommendations for measuring relative cerebral blood volume in brain tumors. Radiology 2008;249:601-13

2. Wetzel SG, Cha S, Johnson G, et al. Relative cerebral blood volume measurements in intracranial mass lesions: interobserver and intraobserver reproducibility study. Radiology 2002;224:797-803
3. Emblem KE, Nedregaard B, Nome T, et al. Glioma grading by using histogram analysis of blood volume heterogeneity from MR-derived cerebral blood volume maps. Radiology 2008;247:808-17

4. Law M, Young R, Babb J, et al. Histogram analysis versus region of interest analysis of dynamic susceptibility contrast perfusion MR imaging data in the grading of cerebral gliomas. AJNR Am J Neuroradiol 2007;28:761-66

5. Lupo JM, Cha S, Chang SM, et al. Dynamic susceptibility-weighted perfusion imaging of high-grade gliomas: characterization of spatial heterogeneity. AJNR Am I Neuroradiol 2005;26:1446-54

6. Otsu NA. A threshold selection method from gray-level histograms. IEEE Trans Syst Man Cybern B Cybern 1979;9:62-66

7. Emblem KE, Due-Tonnessen P, Hald JK, et al. Automatic vessel removal in gliomas from dynamic susceptibility contrast imaging. Magn Reson Med 2009;61:1210-7

8. Boxerman JL, Schmainda KM, Weisskoff RM. Relative cerebral blood volume maps corrected for contrast agent extravasation significantly correlate with glioma tumor grade, whereas uncorrected maps do not. AJNR Am J Neuroradiol 2006;27:859-67 